\section{Still No. 1 for a reason}

Wisdom Toothbrushes is delighted to announce the launch of the brand new Clean Between Pro Interdental Brushes.

Taking interdental cleaning to a new level, the new products feature a uniquely curved profile and broader thumbgrip to improve access to all areas of the mouth. The thicker stem is designed for extra strength and the robust, brush-like tip gently yet effectively sweeps away plaque and bacteria.

Clinically proven to reduce plaque and bleeding, these are the latest generation of the UK's favourite Wisdom Clean Between Interdental Brushes. They come in packs of 30 in the same three sizes as the current brushes - small, medium and large and also now offer a detachable transparent case for the ultimate convenience when storing or travelling.

Wisdom is proud to be the No. 1 selling rubber interdental brush brand, helping patients maintain healthy teeth for life. Discover the new Clean Between Pro Interdental Brushes at www. wisdomtoothbrushes. com or call 01440 714800 .

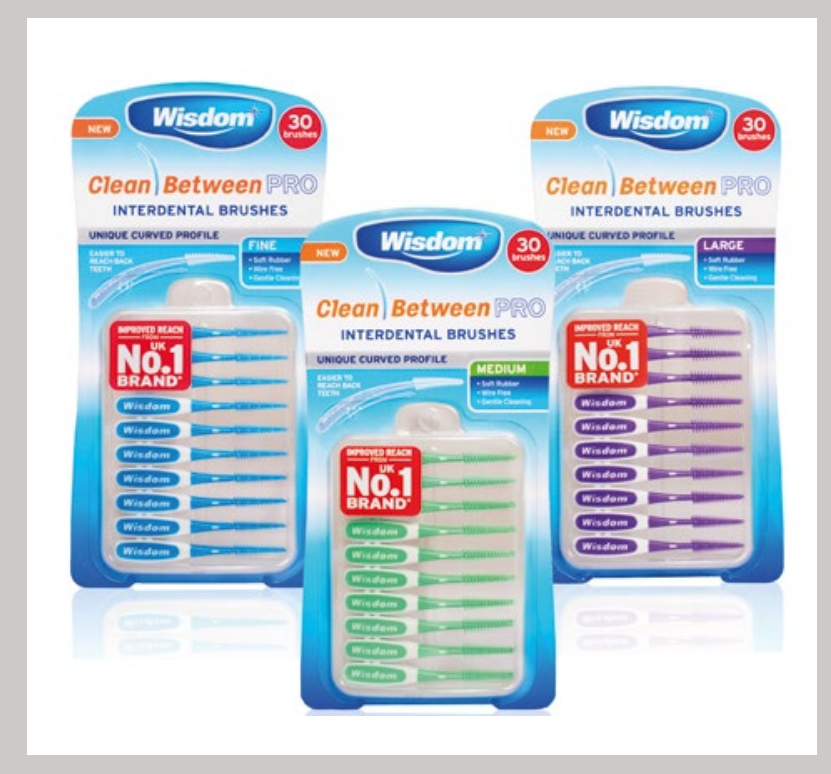

\section{See your career take flight}

The PG Cert in Implant Dentistry from Ucer Education is designed to prepare clinicians for the Diploma in Implant Dentistry from the Royal College of Surgeons of Edinburgh.

The 12-month course offers a formal Level 7 award accredited by EduQual, providing all the theoretical knowledge and hands-on experience needed to place and restore

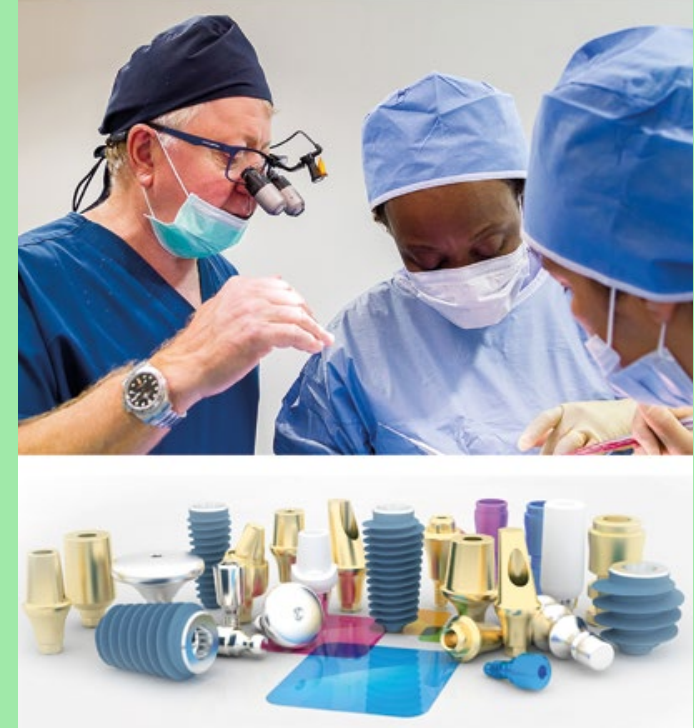
implants confidently.

Only the highest standard evidence-based products are used, including the innovative AnyRidge implant system from MegaGen.

A game-changer for the industry, it is the first system to introduce the concept of a progressive thread design. The training offers delegates the perfect opportunity to try the system out for themselves while gaining practical skills.

For more information on the PG Cert in Implant Dentistry from Ucer Education, please visit https://ucer.education or call 01612371842

\section{Universal glow}

Practitioners need to ensure that all patients have a positive experience in the chair. This is especially important for patients who suffer from dental anxiety or phobia.

Look for the very best materials that allow you to perform treatment efficiently and also gives fantastic results. For restorative work, look no further than the COLTENE product range.

It includes the BRILLIANT EverGlow, an all-round universal composite which offers handling convenience for dentists and high, long-lasting gloss and shade aesthetics for patients. Dentists will find it easy to work with, with good wettability and low stickiness to instruments.

To find out more visit www.coltene. com, emailinfo.uk@coltene.com or call 01444235486.

\section{All under one roof}

Goodman Grant - Lawyers for Dentists has developed an exceptional reputation for offering a broad range of legal services under one roof, catering to the specialist needs of dental professionals across the UK.

With over 100 years of combined experience, Goodman Grant can guide you through dental practice sales and acquisitions, as well as help you develop sound exit strategies that protect your personal and professional future.

Goodman Grant also has a confidential brokerage team who offer valuation and confidential practice brokerage services. They can attend viewings and negotiate the best deal for your sale.

You can also rely on Goodman Grant to provide expert legal support in relation to practice sales and purchases, leases, CQC applications, practice incorporation, partnership and associate agreements, employment contracts, dispute resolution, tendering consortia, drafting legal agreements, and regulatory issues.

For more information visit www. goodmangrant.co.uk or contact your nearest office. 